\title{
Practice Characteristics Associated with Better Implementation of Patient Self-Management Support
}

\author{
Bonnie T. Jortberg, PhD, RD, CDE, Douglas H. Fernald, MA, \\ Danielle M. Hessler, PhD, L. Miriam Dickinson, PhD, Robyn Wearner, RD, MA, \\ Lauri Connelly, MS, Jodi Summers Holtrop, PhD, MCHES, Lawrence Fisher, PhD, \\ and W. Perry Dickinson, MD
}

Background: Advanced primary care models emphasize patient-centered care, including self-management support (SMS). This study aimed to promote the translation of SMS into primary care practices and reported on key baseline practice characteristics that may impact SMS implementation.

Methods: Thirty-six practices in Colorado and California participated in the study from December 2013 to March 2017. Practice administrators completed a Practice Information Form describing practice characteristics. Clinicians and staff $(n=716)$ completed the Practice Culture Assessment and the Patient-Centered Medical Home (PCMH) Monitor. Descriptive statistics were computed to determine practice characteristics related to culture, quality improvement, level of PCMH, and SMS implementation. Field notes and key informant interviews provided contextual details about practices. Iterative qualitative analyses identified important facilitators and barriers and change capabilities around SMS implementation.

Results: In bivariate analyses, rural locations, fewer uncontrolled patients with diabetes, higher Medicaid or uninsured populations, underserved designation, and higher level of "PCMHness" were associated with greater reported implementation of patient SMS (all $P<.05)$ at baseline. In the final multilevel model, specialty (FM vs mixed, $P=.0081)$, rural location $(P=.0109)$, and higher percent Medicaid $(P<.0001)$ were associated with greater SMS. Practices described key facilitators (alignment, motivation, a visible champion, supporting infrastructure, and functional quality improvement and care teams) and barriers (no shared vision, no visible champion, siloed infrastructure, competing programs, turnover, and time constraints) to improving SMS delivery.

Conclusions: Careful attention—and action-on key practice characteristics and context may create more favorable initial conditions for practice change efforts to improve SMS in primary care practices. (J Am Board Fam Med 2019;32:329-340.)

Keywords: California, Chronic Disease, Colorado, Counseling, Disease Management, Information Technology, Life Style, Obesity, Patient Care Team, Patient-Centered Care, Primary Health Care, Quality Improvement, Self Care, Type 2 Diabetes Mellitus

Most patients with type 2 diabetes mellitus (T2DM) in the United States receive diabetes care in primary care settings, which are undergoing rapid transformations due to the need to improve quality and decrease costs. The Patient-Centered

This article was externally peer reviewed.

Submitted 23 April 2018; revised 21 December 2018; accepted 21 December 2018.

From Department of Family Medicine, University of Colorado School of Medicine, Aurora, CO (BTJ, DHF, LMD, RW, LC, JSH, WPD); Department of Family and Community Medicine, University of California, San Francisco, San Francisco, CA (DMH, LF).
Medical Home (PCMH) and the Chronic Care Model are complementary clinical intervention frameworks that are commonly used to support

Funding: none.

Conflict of interest: none declared.

Corresponding author: Bonnie T. Jortberg, PhD, RD, CDE, University of Colorado School of Medicine, Department of Family Medicine, 12631 E. 17th Ave., Mail Stop F496, Aurora, CO (E-mail: bonnie.jortberg@ucdenver.edu).

See Related Article on Page 341. 
better T2DM outcomes in primary care. ${ }^{1-7}$ Selfmanagement support (SMS) is a core component of both the PCMH and Chronic Care Model, and focuses on providing patients with the necessary tools and resources so that they can better manage their illness, in particular by adopting and implementing healthy behaviors that promote optimal clinical outcomes. ${ }^{6,8-10}$ SMS for diabetes typically targets improvements in medication adherence, diet, exercise, and other risk-related behaviors, all which are crucial for maintaining good glycemic control and reducing the risks of diabetes-related complications. Despite its recognized importance and success in clinical trials, SMS programs for diabetes continue to demonstrate limited adoptability and sustainability in the real world of primary care. ${ }^{11,12}$ Primary care clinicians have been unable to comprehensively and consistently address diabetes self-management within an efficient and systematic SMS framework for several interrelated reasons: they are often overwhelmed by competing demands, are poorly trained in assessing and intervening with health behavior change, lack practice systems for implementing change and quality improvement, and receive inadequate reimbursement for time spent in SMS activities. ${ }^{13-16}$

Few tools are available to assist practices with SMS. Interactive behavior-change technology (IBCT) can facilitate the adoption of crucial SMS interventions in primary care for patients with diabetes and related health risk behaviors. ${ }^{17-20}$. Compared with traditional, unstructured programs, technological options for delivery have the advantage of increased convenience and accessibility and may provide individualized support and resources necessary for initiating and maintaining healthful lifestyles, especially when they include nonautomated options to address patient preference and permit patient tailoring. ${ }^{19-22}$ There is strong evidence that Internet-based programs can effectively promote health behaviors to support diabetes selfmanagement, ${ }^{23}$ such as healthful eating/weight management, ${ }^{24-27}$ increasing physical activity, ${ }^{28-30}$ reducing depression symptoms, and smoking cessation. ${ }^{31,32}$ Multiple randomized trials have been conducted using IBCT programs for diabetes selfmanagement with positive results. ${ }^{33,34}$

Connection to Health (CTH) is a comprehensive, evidence-based SMS program that supports behavior change through IBCT. The CTH logic model is informed by social-cognitive $e^{35-37}$ and so- cial-ecological $^{38-40}$ theories and is inclusive of the evidence based principles for implementing SMS in primary care. ${ }^{41}$ Multiple intervention components work together to promote enhanced, tailored diabetes management, which is linked to positive health outcomes. ${ }^{42-45}$

The simple availability of effective IBCT tools such as CTH does not assure their successful implementation. ${ }^{15,16,46-48}$ Primary care practices are experiencing multiple pressures to see a large number of patients, to provide improved care, and to do so with very constrained reimbursement. Practices have few mechanisms to integrate new programs into routine care, which can exert major pressures on practice operations-even small changes can have substantial consequences that limit their effectiveness and sustainability. ${ }^{15,16,46-48}$ Adoption and implementation of new care programs vary across practices based on practice characteristics, including practice culture and change capacity, practice size, location (rural vs urban), previous change experience, and decision-making style. ${ }^{13,14,46}$

This study was designed to promote the translation of SMS into primary care practices for patients with T2DM by combining 2 promising lines of research; specifically, testing the effectiveness of CTH for patients with T2DM in diverse primary care practices and evaluating the impact of practice facilitation to enhance uptake and maintenance of the intervention over time. This article reports on the baseline quantitative and qualitative results, which address this specific aim: to identify key practice characteristics (eg, practice size, organization, setting, and level of experience with practice redesign efforts) that affect CTH implementation and outcomes. The 2 other specific aims of this study, which will be reported on in another manuscript, are (1) to conduct a cluster randomized trial to examine the reach, effectiveness, adoption, implementation, and maintenance of CTH for patients with T2DM in primary care practices; and (2) to determine the incremental benefit of brief, targeted practice facilitation on the implementation of $\mathrm{CTH}$ in diverse primary care practices. ${ }^{49}$

\section{Methods and Design \\ Design}

CTH is a 3 -arm, cluster-randomized trial to evaluate the reach, effectiveness, adoption, implementation, and maintenance of CTH for patients with 
T2DM in diverse primary care practices. This report is focused on postrandomization practice and practice member surveys and key informant interviews collected at baseline, before any intervention implementation.

\section{Practice Sample}

We recruited 36 primary care practices, 18 each in Colorado and California. Practices were recruited through the Colorado and California practicebased research networks, informational articles in the Colorado Academy of Family Physicians quarterly newsletter, and direct investigator contacts with health systems and individual practices. Inclusion criteria were family medicine or general internal medicine practices with a minimum of $80 \mathrm{pa}-$ tients with T2DM, with all clinicians agreeing to participate. Practices were selected to participate if they met these criteria. We recruited a diverse set of practices of various sizes and organizational structures (such as private, system-owned, and safety-net practices). Detailed information about practice recruitment was published in a previous manuscript. ${ }^{50}$ Practices participated for 18 months, with participation encompassing December 2013 to March 2017.

\section{Protections}

This project was reviewed and approved by the Colorado Multiple Institutional Review Board and the University of California San Francisco Institutional Review Board.

\section{Practice Participants}

All practice staff and clinicians were asked to complete the baseline surveys, resulting in 716 completed surveys from all 36 practices. In addition, 3 to 6 staff and clinicians per practice participated in key informant interviews. Interviewees were selected to represent a mixture of roles in the practice (ie, clinical and practice leadership, nursing, front and back office staff, and health educators) to ensure capture of the breadth of experience related to SMS.

\section{Measures}

\section{Practice Surveys}

Clinicians and staff completed 2 surveys to determine baseline practice characteristics related to culture, quality improvement, PCMH-ness, and SMS implementation:
(1) The SMS subscale of the Practice Monitor provided information about clinician and staffs' routine use of SMS as a component of patient care. Practice members rated their perceptions of SMS activities and practice culture using a Likert scale (strongly disagree to strongly agree) on 28 items. Prior validation and factor analysis yielded 7 reliable subscales used in our analysis; this survey can be found in Appendix A online.

(2) The Practice Culture Assessment (PCA) provided practice-wide information about practice culture related to practice change and improvement. ${ }^{50}$ The PCA was completed by practice clinicians and staff using a 5-point Likert-type scale (strongly disagree to strongly agree) on 22 items. Before validation and factor analysis yielded 3 reliable subscales used in our analysis: improvement and Change Culture, Work Relationships, and Chaos. ${ }^{51}$

In addition, practice administrators completed a Practice Information Form, a 16-item form that describes main practice characteristics and descriptions of their patient population.

\section{Survey Data and Statistical Analysis}

Surveys were administered from introduction through the first SMS training session. Practice staff and clinicians completed the survey either via article copy or through an email link to Research Electronic Data Capture, which is a secure, webbased application designed to support data capture for research studies. ${ }^{52}$ Surveys were anonymous, and no individual information was obtained or shared with other staff.

Practice characteristics were collected during the practice recruitment process. Practice characteristic data collected were level of quality improvement experience, level of PCMH implementation, number of clinicians, specialty type, percent of patients with diabetes, percentage of patients with hemoglobin A1c >9, location (rural/urban), type of practice organization, and percentage of Medicaid or uninsured patients.

\section{Survey Data Analysis}

Descriptive statistics were computed for baseline practice characteristics and key variables of interest from the practice member surveys. Exploratory factor analysis and item analysis were performed for items the SMS scale from the Practice Monitor survey, because this specific combination of items had not been previously used as a scale. The out- 
Figure 1. Framework for self-management support (SMS) practice improvement. Adapted from: Solberg LI. Improving medical practice: a conceptual framework. Ann Fam Med 2007;5:251-256.

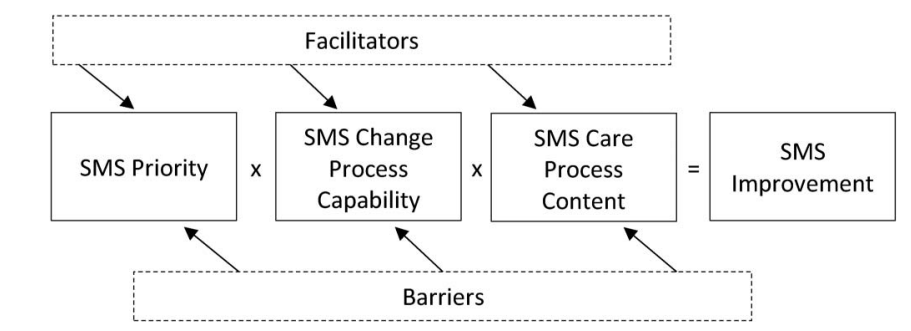

Adapted from: Solberg LI. Improving medical practice: a conceptual framework. Ann Fam Med. 2007;5(3):251-256.

come variable for all subsequent analyses was the patient SMS scale. Independent variables of interest included items from the practice survey, listed above, and scores on the PCA subscales Change Culture, Chaos, and Work Culture. Each independent variable was assessed for possible association with the outcome in bivariate analyses (Pearson correlation coefficient); practice characteristic variables that were associated with the outcome at $P<$ .10 in simple bivariate associations were considered for further analysis; PCA subscales were considered for further analysis regardless of strength of bivariate associations. Next, general linear mixed models (with random intercept for practice) were used to further examine associations between the outcome and each practice-level variable, 1 at a time. Practice-level variables that were significantly associated with the outcome $(P<.05)$ after adjusting for clustering were included in an initial multivariable model, eliminating nonsignificant variables 1 at a time until all $P$ values were less than 0.05 . Finally, practice culture variables were added to the models. Because of collinearity, Change Culture and Work Culture were assessed in separate models. All statistical analyses were performed using SAS version 9.4 (SAS Institute Inc., Cary, NC).

\section{Qualitative Data}

Baseline qualitative data included (1) structured field notes completed by the facilitator and/or research staff after each substantive contact with the practice (from introduction through the first SMS training session) and (2) semistructured interviews conducted with staff and clinicians in each practice (before or immediately after the first SMS training session). Memo forms providing brief summary notes by the interviewer were also completed after each interview. ${ }^{53}$ Over 250 documents were included in this analysis.

\section{Qualitative Data Analysis}

Four qualitative analysts followed an iterative approach to the qualitative analysis, with the investigators going through cycles of reading, summarizing, and rereading the data. ${ }^{54}$ Beginning with immersion in the data (each analyst reviewed data from a selection of practices in each Colorado and California and in each arm) to read, review, and reread, and then to the development of conceptual categories relevant to the implementation of CTH or SMS. Fourteen provisional conceptual categories emerged, which were then used for template coding to begin segmenting relevant data for further review and analysis. After a review of coded data to assure conceptual inter-rater reliability, the analysts divided the data and coded it using the qualitative software program ATLAS.ti (version 7, Scientific Software Development, GmbH). The analysts continued to meet and discuss the data over a series of twice-monthly meetings over 6 months. Reading, refining, discussing, and summarizing continued until key themes emerged and clarity by practice for each theme was evident. The coded data were then summarized into a matrix, with key practice characteristics organized into the Solberg framework for practice improvement (see Figure 1). Finally, the analysts developed practice characteristic constructs as forces that potentially propel or repel the implementation of SMS in these practices.

\section{Results}

\section{Practice Survey Results}

A total of 716 clinicians and staff enrolled in CTH completed baseline surveys (73\% response rate). Practice characteristics are shown in Table 1. The majority of the practices enrolled in CTH were Federally Qualified Health Centers, and as such, the percentage of patients with Medicaid or uninsured is higher than in the general population. 
Table 1. Baseline Practice Characteristics from 36 Primary Care Practices in Colorado and Northern California; Data Collected from 12/2013 to 9/2015

\begin{tabular}{lcc}
\hline Variables & Mean (SD) or \% & Minimum, Maximum \\
\hline Number of clinicians & $6.86(3.95)$ & 2,16 \\
Specialty (\% FM alone) & $63.9 \%$ & - \\
Type of practice organization (CHC vs other) & $75.0 \%$ & - \\
Number of patients with type 2 diabetes & $508(369)$ & 60,1542 \\
Percentage of diabetic patients with HgAlc $>9$ & $26.7(8.8)$ & $4.3,46.0$ \\
Percentage Medicaid & $38.3(20.2)$ & $0,70.0$ \\
Percentage uninsured & $27.0(8.8)$ & $0.9,56.0$ \\
Percentage rural location & 27.8 & - \\
Percentage care manager present & 27.8 & - \\
Ongoing Quality improvement team meets regularly, & $89.9 \%$ & - \\
$\quad$ with or without outside assistance & $66.7 \%$ & - \\
NCQA PCMH recognition & &
\end{tabular}

FM, family medicine; CHC, Community Health Center; NCQA, National Committee for Quality Assurance; PCMH, PatientCentered Medical Home; SD, standard deviation.

Overall scores on practice member surveys for patient SMS activities and practice culture are shown in Table 2. Patient SMS Cronbach's $\alpha=$
0.92. The overall score for patient self-management was 65.7 out of 100 , and individual SMS items showed slightly higher implementation of

Table 2. Baseline Practice Member Surveys from 36 Primary Care Practices in Colorado and Northern California; Data Collected from 12/2013 to $9 / 2015$

\begin{tabular}{|c|c|c|c|}
\hline Name of Survey & Measure & Items & Mean (SD) \\
\hline \multirow[t]{8}{*}{ Practice Monitor } & Patient SMS (overall score) & & $65.7(20.9)$ \\
\hline & $\begin{array}{l}\text { SMS individual items ( } 0 \text { to } \\
5 \text { range) }\end{array}$ & $\begin{array}{l}\text { System implemented for identifying patient needs for } \\
\text { assistance with psychosocial issues, health behavior } \\
\text { change, and managing chronic illness }\end{array}$ & $3.45(1.19)$ \\
\hline & & $\begin{array}{l}\text { System implemented for assisting patients with } \\
\text { developing shared care plans with specific goals } \\
\text { and action plans for health behavior change and } \\
\text { management of chronic illness }\end{array}$ & $3.25(1.25)$ \\
\hline & & $\begin{array}{l}\text { Shared care plans are developed collaboratively with } \\
\text { patients and families }\end{array}$ & $3.14(1.30)$ \\
\hline & & $\begin{array}{l}\text { Care plans and action plans are regularly reviewed to } \\
\text { monitor patient progress in accomplishing goals }\end{array}$ & $3.11(1.30)$ \\
\hline & & $\begin{array}{l}\text { Patients and families are actively linked with } \\
\text { community resources to assist with their self- } \\
\text { management goals }\end{array}$ & $3.20(1.25)$ \\
\hline & & $\begin{array}{l}\text { Patients and families are provided with tools and } \\
\text { resources to help them engage in the management } \\
\text { of their health between office visits }\end{array}$ & $3.40(1.21)$ \\
\hline & & $\begin{array}{l}\text { There is a care manager or other staff members in } \\
\text { the practice trained to assist patients and families } \\
\text { in health behavior change and chronic disease } \\
\text { management }\end{array}$ & $3.43(1.45)$ \\
\hline $\begin{array}{l}\text { Practice Culture } \\
\text { Assessment }\end{array}$ & Change Culture & & $64.0(18.9)$ \\
\hline $\begin{array}{l}\text { Practice Culture } \\
\text { Assessment }\end{array}$ & Work Culture & & $63.8(18.4)$ \\
\hline $\begin{array}{l}\text { Practice Culture } \\
\text { Assessment }\end{array}$ & Chaos & & $43.5(18.5)$ \\
\hline
\end{tabular}

$\mathrm{N}=716$ staff and clinicians. SD, standard deviation; SMS, self-management support. 


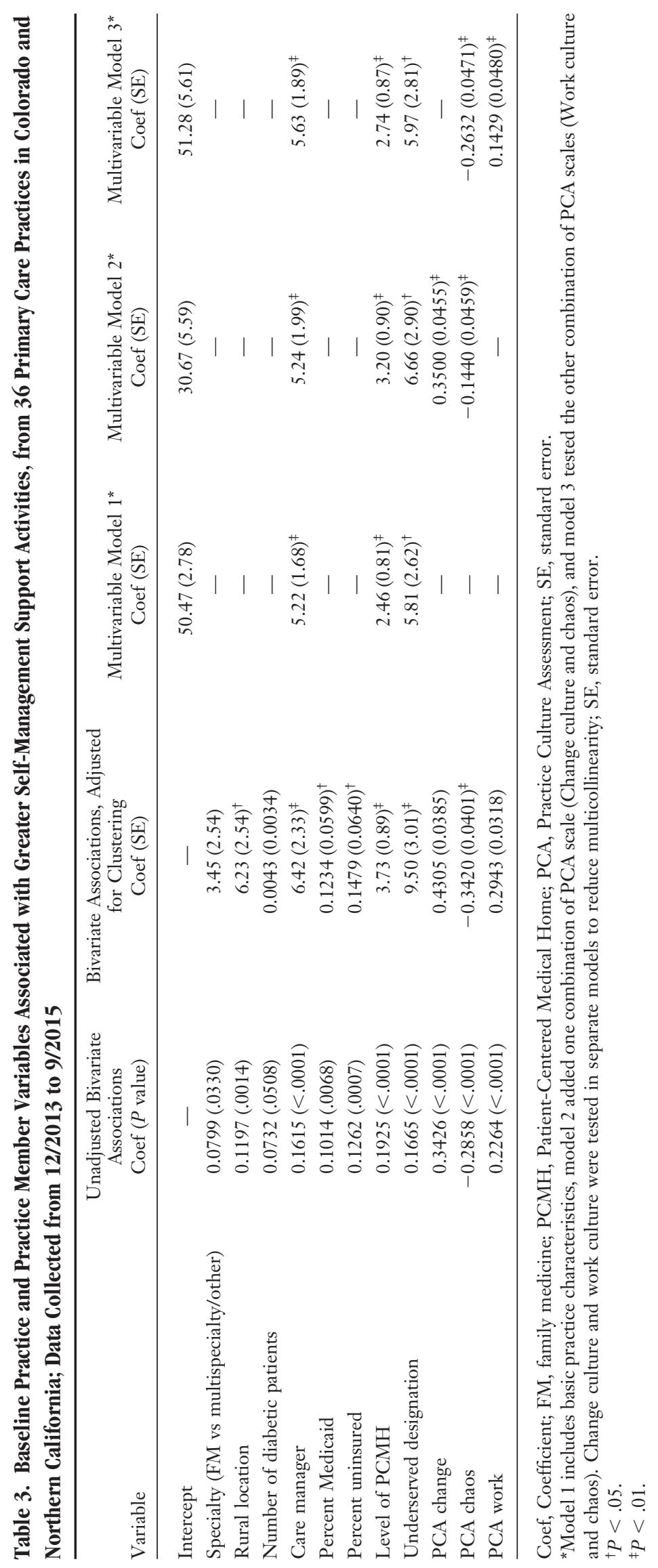


Figure 2. Facilitators and barriers for self-management support (SMS) implementation.

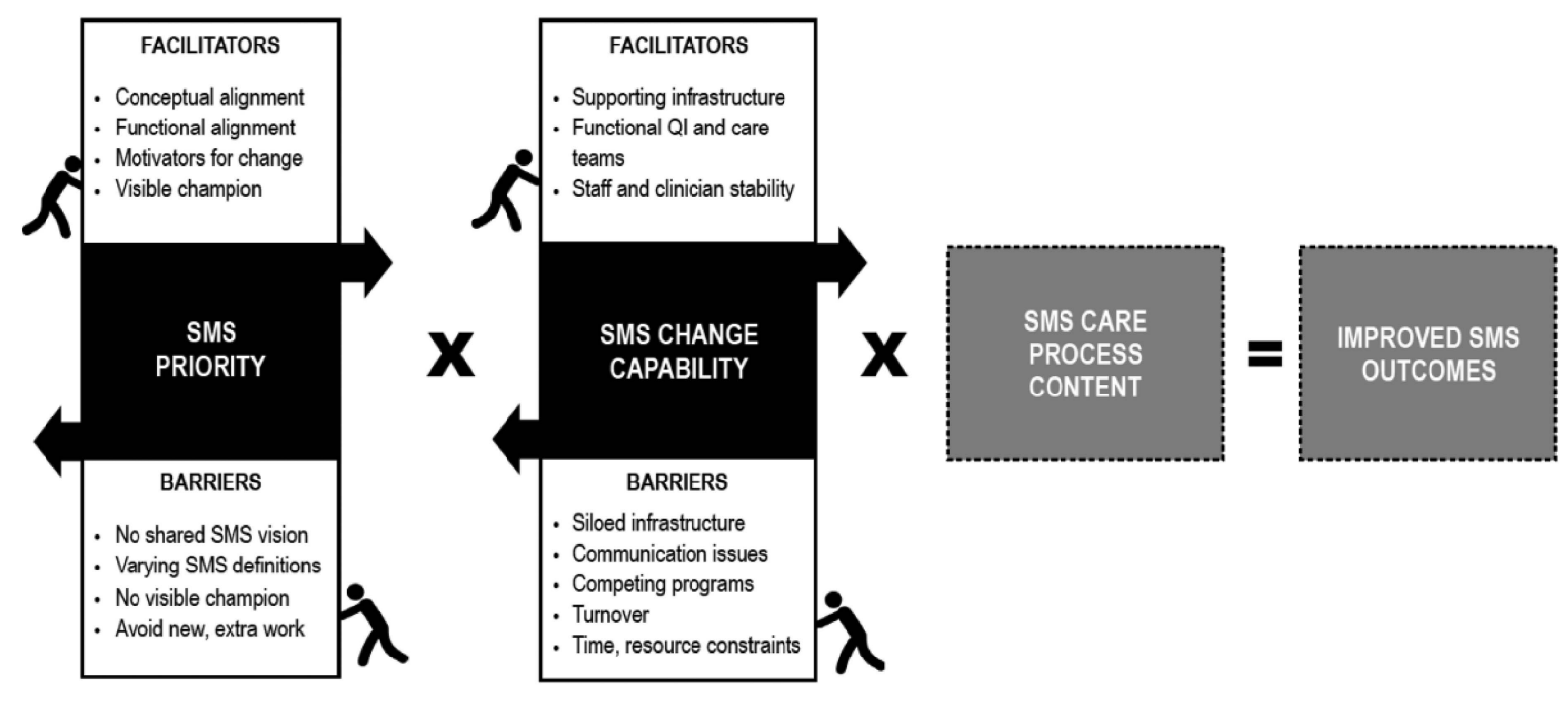

specific SMS activities, such as systems implemented for identifying patients with needs for SMS, use of shared care plans, SMS tools provided to patients and families, and the presence of a care manager or staff member to assist with SMS activities.

Simple bivariate associations for variables that met initial screening criteria are shown in Table 3. After adjusting for clustering, the following variables were associated with a greater reported implementation of patient SMS (all $P<.05$; please see Table 2 for specific SMS items): rural location, higher percent Medicaid or uninsured populations, underserved designation, presence of a care manager, and a higher level of "PCMHness" (see Table 2). In the multivariable model with SMS implementation as the outcome and all practice level variables from the previous step included initially as independent variables, presence of a care manager $(P=.0019)$, level of "PCMHness" $(P=.0027)$, and underserved designation $(P=.0272)$ were retained at $P<.05$. Because of high collinearity between PCA subscales Change Culture and Work Culture, these were included in separate models. After adjusting for significant practice level variables, higher scores on the Change Culture $(P<.0001)$ and Work Culture $(P=.0030)$ subscales were associated with greater SMS. Higher scores on the Chaos $(P=.0020)$ subscale was associated with lower scores on SMS implementation.

\section{Qualitative Results}

Across all 36 practices, 119 baseline key informant interviews were completed (42 providers; 77 staff or administrators) and 53 field notes were filed covering the baseline period. Delivering SMS likely requires multiple people in a clinic having active and integrated roles in delivering key components of SMS to patients. Leif Solberg's work on practice improvement provided an organizing framework to describe initial conditions that could potentially promote or hinder the changes needed to effectively deliver SMS for patients with diabetes (Figure 2). The baseline analysis focused on Priority and Change Process Capability to elaborate details in the "black box" of these areas. It also provided a conceptual framework to organize analytic conceptual constructs over time. From the analysis of baseline data, practice priorities were identified and change process capabilities that could affect SMS implementation in these 36 practices (Figure 2).

\section{Priority: Alignment Around SMS Transformation}

At baseline, across all 36 practices, there were clinical leaders, staff, and clinicians who expressed SMS transformation as an important priority; however, other practice staff and clinicians expressed simultaneous concerns and reservations about practice changes for SMS. Propelling and repelling factors pushing for SMS transformation are described in Table 4. 


\section{Propelling factors pushing for SMS transformation}

Conceptual alignment: positive emotional response to the principles of SMS; good fit with other quality programs and initiatives.

Functional priority alignment: at least partial fit with improving existing team-based care process priorities, such as group visits, care management, and patient health educators.

Motivation for change and improvement: most clinician leaders expressed desire to improve their SMS delivery, especially in terms of more consistency.

Visible champions: in about half of the practices, there was at least one individual (clinician, staff, or administrator) who showed interest in committing effort to SMS changes.

Repelling factors pushing against SMS transformation

No shared vision for how SMS aligns with practice priorities: The vision for how SMS aligns conceptually or functionally was rarely a vision shared or discussed among all staff, clinicians, or administrators. There were early concerns about full buy-in across staff and providers, especially that the processes, staff roles, time, and resources would be insufficient. Reluctance, hesitance, or passive interest in SMS was especially visible among staff in meetings we observed.

Varying definitions of SMS: How staff and clinicians described SMS often included some components (e.g., goal-setting, action plans, collaborative decisions), but rarely were these complete or shared across staff and clinicians. In several cases, there were perceptions that SMS was already done by someone else in the practice.

No visible champion for SMS changes: In about half of the practices there were no staff or clinicians who were visible champions for SMS changes or improvements.

Priority on avoiding adding more work and more duties: In most practices, there were concerns about already being busy with current work. Clinicians were concerned about extra time needed with patients or for documentation; staff, especially health educators, expressed concerns about adding extra duties, or duplicated work, or extending beyond their job descriptions.
"I have a group of patients in my practice who are pretty controlled but they once in a while will get out of control. Having some type of selfmanagement support that would help them to figure out, 'What am I doing wrong so I can get back on track,' would be helpful."

"The clinicians who are now doing all of the SMS said that it usually gets pushed into the last 2 minutes of an appointment, when it gets covered at all. They did seem to think that CTH tools will belp structure and streamline SMS, however."

"Given our population, given the type of constraints but I'm really excited to be able to track whether it's making a difference because we've had diabetes clinic for quite some time. I've always been curious to know well how have those patients who have had those three professions intervention have they done? We have seen some changes, but I've not been able to gather that information and kind of present it in a way that it shows it's made a difference."

"My impression is that they can absolutely do something with CTH because the PA wants to try. She stayed after the training to ask questions about the time commitment and other things."

"A [practice] manager [said] that this practice is 'not a strong team' prior to the training session. They 'just get things done' and historically have operated in 'survivorship' mode. ... Both providers were present and one of them. . came armed with a lot of implementation questions. . she was engaged. A comment from [another provider] today signaled impatience as she wanted a quick decision, 'Can we just make a decision and stop talking about it.'”

"[SMS means] That they take care of their own selves with managing their own diabetes or whatever it is; to manage their own care and to know what goal they had for the next month. Fust to take care of their own self. "[SMS] means, starting with the patient and asking what they need and giving recommendations or maybe more importantly, collaborating with the patient on strategies to address what they think they need. Telling them what to do, based on what the PCP thinks they need.

"She [care coordinator] doesn't identify a strong SMS champion or "driver" at the clinic at this time. She doesn't think there is a shared approach or structure re SMS that is used across all providers/ care teams."

"The clinician who was most actively engaged during the presentation talked with me afterward, saying that she thinks the staff is passive and it may therefore be challenging to get SMS up and running practicewide. . . based on the staff's very limited response to the presentation, it appears likely that they would be willing to do SMS work as assigned by leadership, but probably would not volunteer for expanded roles with patients or be assertive with recommendations for SMS implementation"

CTH, Connection to Health.

\section{Change Process Capability to Implement SMS Changes}

The details of change process capability varied widely across the 36 practices; however, there were consistent concepts that emerged about characteristics that propel implementation-supporting infrastructure, functional teams, and practice stability - and those that repel implementation-siloed infrastructure, communication issues, turnover, competing programs, and time/resource constraints. Propelling and repelling factors for change process capabilities are described in Table 5 .

\section{Discussion}

The purpose of this article was to report on baseline practice characteristics and context from surveys and qualitative interviews that reflect previous implementation of SMS activities before participation in this study. SMS is a critical element of care for people with diabetes and 
Propelling factors pushing for change process capabilities

Supporting infrastructure: Most practices at baseline had at least some resources, workflows, visits, and roles already deployed that aligned with delivering more robust patient SMS, such as, patient health educators/ care coordinator, group visits, or existing workflows.

Functional Care Teams or QI Teams: At least moderately functional teams (QI or care teams) were observed in some practices where clinicians, staff, and administrators were present and at least minimally engaged in some decision making around SMS changes; or they appeared to have systems in place to communicate across care team roles or QI teams about changes planned.

Staff and clinician stability: There were several practices that described staff and clinicians who had been with the practice and for multiple years, and had good knowledge of how the clinic operates and experience with practice change or quality improvement.

Repelling factors pushing against change process capabilities

Siloed infrastructure: Especially, in larger practices, clinical roles were siloed with integration across roles beyond an initial referrals or hand-offs. Most practices appeared to have ad hoc approaches to quality improvement work. Among those in systems, there was no specific mention of how the systems would directly support the QI processes at the practice level.

Communication issues: Communication across roles or from systems leaders to practices was incomplete. This extended to how decisions were made, with staff and clinicians in several clinics feeling that the decision to participate was "made for us" by someone else in their system.

Time and resource constraints: The capacity to take on additional work, was acknowledged widely among the clinical leadership as a potential threat to fully implementing SMS. There were numerous concerns raised about capacity of clinicians-and especially staff-to take on new work or different work, change workflows, and adopt new tools.

Potential turnover/arrival of key people: Even at baseline, eight practices described the recent arrival or anticipated departure of key clinicians, including medical directors, SMS champions, diabetes clinic directors, or behavioral health providers.

Competing programs: Just a few practices made notable mentions, at baseline, of the potential for competing programs to affect implementation of CTH/SMS.
"'I found this team attentive and engaged in the topic.... I was struck by the empowered role of the MAs here. They do so much more than room patients take vital signs and follow the clinician's directions.... I see the benefits as two-fold: the MAs seem happy and engaged and the clinicians don't appear stressed. Their workflow is working for them." [FN 110]."

"The team already has many of the attitudes and skills (follow up calls to check on action plan progress, etc.) that will belp them be successful in expanding SMS with their patients." [MF 202]

"After 24 years in this small clinic, she [practice manager] is well aware of every aspect of the practice. It is a very pleasant practice. She understands practice redesign. [MF 116" [MF 202]

"I don't cross paths with the PHEs." [BHP 110]

"I don't know exactly what the providers do about SMS." [PHE 110]

"Does not feel diabetes care is collaborative. The lack of collaboration is a "company issue" because it "takes time and providers slow down". [FN clinic 110]

"She noted that the team is "not quite as knit as it should be" because the provider and their $M A$ "circle" but the rest of the team may not know due to "limited communication." [MF clinic 110]

"She noted that the team is "not quite as knit as it should be" because the provider and their $M A$ "circle" but the rest of the team may not know due to "limited communication." [MF clinic 110]

"A new development was that our previous contact, a PA, is leaving [the practice]. This person was also the lead clinician for the Shared Medical Appointments that [practice] is putting stock into to belp manage the diabetes populations for each clinician." [FN clinic 101]

"They seem to bave a lot going on. A new QI tracking program that is discussed in the PA interview, a mandated state Wise Women's Program and now this project." [FN, 104]

CTH, Connection to Health.

other chronic conditions, and the current findings may assist primary care practices and researchers with identifying key practice characteristics that are necessary to provide SMS for patients with T2DM.

At baseline, the practices reporting higher levels of SMS reported significantly higher levels of a positive work culture, the presence of a care manager, higher levels of "PCMHness," and lower levels of practice Chaos. These characteristics are known to cluster and are often seen together, as have been reported in other studies. ${ }^{48,53}$ For example, the Enhancing Practice, Improving Care study found that practice facilitation using a continuous quality improvement approach was effective at improving measures of diabetes care. ${ }^{51}$ Likewise, Shetty et $\mathrm{al}^{55}$ examined the components of organizational support for SMS in primary care practices. These researchers found 8 essential elements of organizational support, including ongoing quality 
improvement, staff training and education, and integration of SMS into primary care.

Results from the qualitative data also support the findings from the practice surveys regarding level of "PCMH-ness" and the importance of the presence of a care manager. SMS implementation facilitators included several key components of the $\mathrm{PCMH},{ }^{1}$ including functional quality-improvement teams; coordinated care with the presence of supporting infrastructure such as care managers, patient health educators, or Certified Diabetes Educators; and practice leadership who saw alignment with SMS and their PCMH activities.

Leif Solberg's quality improvement framework suggests that multiple factors combine to produce improved quality. SMS is not business as usual. Results from this study suggest that SMS may require substantial redesign of workflows, responsibilities, and staffing along with careful coordination and communication across multiple roles in a practice for a sustained period. There are factors that seem to align with and promote SMS redesign (eg, conceptual and functional alignment, functional teams, and supporting infrastructure); yet, there may be specific repelling factors (eg, no visible champions, lack of consistent vision, different understandings of SMS, and siloed work) that are particularly at odds with implementing sustained and functional SMS. Addressing and minimizing the repelling forces and maximizing and building on the propelling forces across the entire practice may help to unify the practice vision and point staff and clinicians in the same direction.

These findings may be useful for health systems, practices, researchers, and others to consider in identifying practice's readiness for SMS implementation. Lehman et $\mathrm{al}^{55}$ assessed organizational readiness for change in health care and found similar results, particularly for the importance of a positive work culture to be "ready" to implement technology and organizational changes. As such, it may be useful for practices and health care organizations to assess their work culture before embarking on implementing SMS as foundational work may need to be conducted to ensure success.

\section{Limitations}

This project was only implemented in primary care practices in Colorado and California and may not be representative of primary care in other states. In addition, federally qualified health centers were overrepresented in our sample. Because this is a limited sample, other important factors may exist, but we did not observe them in our data. This report does not consider the relative influence on the observed characteristics on important, downstream practice and patient outcomes.

\section{Conclusion}

Fully implemented SMS requires robust and active participation across clinical roles in primary care practices. The results from this study add to our understanding that careful attention-and action-on work culture and transformation readiness may create more favorable initial conditions for practice change efforts to improve SMS in primary care practices. Practices, health systems, and reseachers may need to take these key practice characteristics into account when considering implementation of SMS targeted for patients with T2DM.

To see this article online, please go to: http://jabfm.org/content/ 32/3/329.full.

\section{References}

1. Joint Principles of the Patient-Centered Medical Home Secondary Joint Principles of the PatientCentered Medical Home 2007. Available from: https://www.aafp.org/dam/AAFP/documents/ practice_management/pcmh/initiatives/PCMHJoint. pdf. Published March 2007. Accessed March 1, 2018.

2. Wagner EH, Austin BT, Von Korff M. Improving outcomes in chronic illness. Manag Care Q 1996;4: $12-25$.

3. Wagner EH, Austin BT, Von Korff M. Organizing care for patients with chronic illness. Milbank Q 1996;74:511-44.

4. Bodenheimer T, Wagner EH, Grumbach K. Improving primary care for patients with chronic illness. JAMA 2002;288:1775-9.

5. Bodenheimer T, Wagner EH, Grumbach K. Improving primary care for patients with chronic illness: the chronic care model. Part 2. JAMA 2002; 288:1909-14.

6. Berenson RA, Hammons T, Gans DN, et al. A house is not a home: keeping patients at the center of practice redesign. Health Aff (Project Hope) 2008; 27:1219-30.

7. Grumbach K, Bodenheimer T. A primary care home for Americans: putting the house in order. JAMA 2002;288:889-93.

8. Riley KM, Glasgow RE, Eakin EG. Resources for health: a social-ecological intervention for supporting self-management of chronic conditions. J Health Psychol 2001;6:693-705. 
9. Glasgow RE, Toobert DJ, Hampson SE. Effects of a brief office-based intervention to facilitate diabetes dietary self-management. Diabetes Care 1996;19: 835-42.

10. Barrera M, Jr., Toobert DJ, Angell KL, Glasgow RE, Mackinnon DP. Social support and social-ecological resources as mediators of lifestyle intervention effects for type 2 diabetes. J Health Psychol 2006;11:483-95.

11. Schillinger D, Handley M, Wang F, Hammer H. Effects of self-management support on structure, process, and outcomes among vulnerable patients with diabetes: a three-arm practical clinical trial. Diabetes Care 2009;32:559-66.

12. Norris SL, Engelgau MM, Narayan KM. Effectiveness of self-management training in type 2 diabetes: a systematic review of randomized controlled trials. Diabetes Care 2001;24:561-87.

13. Tallia AF, Stange KC, McDaniel RR, Jr., Aita VA, Miller WL, Crabtree BF. Understanding organizational designs of primary care practices. J Healthc Manag 2003;48:45-59; discussion 60-1.

14. Solberg LI, Brekke ML, Fazio CJ, et al. Lessons from experienced guideline implementers: attend to many factors and use multiple strategies. Jt Comm J Qual Improv 2000;26:171-88.

15. Jaen CR. Successful health information technology implementation requires practice and health care system transformation. Ann Fam Med 2011;9: 388-9.

16. Sittig DF, Ash JS. On the importance of using a multidimensional sociotechnical model to study health information technology. Ann Fam Med 2011; 9:390-1.

17. Glasgow RE, Bull SS, Piette JD, Steiner JF. Interactive behavior change technology. A partial solution to the competing demands of primary care. Am J Prev Med 2004;27:80-7.

18. Glasgow RE. Interactive media for diabetes selfmanagement: issues in maximizing public health impact. Med Decis Making 2010;30:745-58.

19. Oenema A, Brug J, Dijkstra A, de Weerdt I, de Vries H. Efficacy and use of an internet-delivered computer-tailored lifestyle intervention, targeting saturated fat intake, physical activity and smoking cessation: a randomized controlled trial. Ann Behav Med 2008; 35:125-35.

20. Prochaska JO, Velicer WF, Redding C, et al. Stagebased expert systems to guide a population of primary care patients to quit smoking, eat healthier, prevent skin cancer, and receive regular mammograms. Prev Med 2005;41:406-16.

21. Vandelanotte C, De Bourdeaudhuij I, Brug J. Twoyear follow-up of sequential and simultaneous interactive computer-tailored interventions for increasing physical activity and decreasing fat intake. Ann Behav Med 2007;33:213-9.
22. Glasgow RE, Edwards LL, Whitesides H, Carroll N, Sanders TJ, McCray BL. Reach and effectiveness of DVD and in-person diabetes self-management education. Chronic Ill 2009;5:243-9.

23. Rabin B, Glasgow RE. Dissemination and implementation of interactive health communication applications. New York, NY: Routledge; 2012.

24. Brug J, Oenema A, Campbell M. Past, present, and future of computer-tailored nutrition education. Am J Clin Nutr 2003;77:1028s-34s.

25. Tate DF, Jackvony EH, Wing RR. Effects of Internet behavioral counseling on weight loss in adults at risk for type 2 diabetes: a randomized trial. JAMA 2003;289:1833-6.

26. Oenema A, Brug J, Lechner L. Web-based tailored nutrition education: results of a randomized controlled trial. Health Educ Res 2001;16:647-60.

27. Vandelanotte C, De Bourdeaudhuij I, Brug J. Acceptability and feasibility of an interactive computertailored fat intake intervention in Belgium. Health Promot Int 2004;19:463-70.

28. Wanner M, Martin-Diener E, Braun-Fahrlander C, Bauer G, Martin BW. Effectiveness of active-online, an individually tailored physical activity intervention, in a real-life setting: randomized controlled trial. J Med Internet Res 2009;11:e23.

29. Spittaels H, De Bourdeaudhuij I, Brug J, Vandelanotte C. Effectiveness of an online computer-tailored physical activity intervention in a real-life setting. Health Educ Res 2007;22:385-96.

30. Steele R, Mummery WK, Dwyer T. Using the Internet to promote physical activity: a randomized trial of intervention delivery modes. J Phys Act Health 2007;4:245-60.

31. Strecher VJ, McClure J, Alexander G, et al. The role of engagement in a tailored web-based smoking cessation program: randomized controlled trial. J Med Internet Res 2008;10:e36.

32. Strecher VJ, McClure JB, Alexander GL, et al. Webbased smoking-cessation programs: results of a randomized trial. Am J Prev Med 2008;34:373-81.

33. Austin Boren S, Gunlock TL, Krishna S, Kramer TC. Computer-aided diabetes education: a synthesis of randomized controlled trials. AMIAAnnu Symp Proc 2006:51-5.

34. Welch G, Weinger K, Anderson B, Polonsky WH. Responsiveness of the Problem Areas In Diabetes (PAID) questionnaire. Diabet Med 2003;20:69-72.

35. Bandura A. Self-efficacy: The exercise of control. New York, NY, US: W H Freeman/Times Books/ Henry Holt \& Co; 1997.

36. Glasgow RE. A practical model of diabetes management and education. Diabetes Care 1995;18:117-26.

37. Glasgow RE, Eakin EG. Issues in diabetes self-management. New York: Springer Publishing Company; 1998.

38. Glasgow RE, Strycker LA, Toobert DJ, Eakin E. A social-ecologic approach to assessing support for dis- 
ease self-management: the Chronic Illness Resources Survey. J Behav Med 2000;23:559-83.

39. McLeroy KR, Bibeau D, Steckler A, Glanz K. An ecological perspective on health promotion programs. Health Educ Q 1988;15:351-77.

40. Stokols D, Allen J, Bellingham RL. The social ecology of health promotion: implications for research and practice. Am J Health Promot 996;10:247-51.

41. Battersby M, Von Korff M, Schaefer J, et al. Twelve evidence-based principles for implementing selfmanagement support in primary care. Jt Comm J Qual Patient Saf 2010;36:561-70.

42. Daubenmier JJ, Weidner G, Sumner MD, et al. The contribution of changes in diet, exercise, and stress management to changes in coronary risk in women and men in the multisite cardiac lifestyle intervention program. Ann Behav Med 2007;33:57-68.

43. Ades PA. Cardiac rehabilitation and secondary prevention of coronary heart disease. N Engl J Med 2001;345:892-902.

44. Brochu M, Poehlman ET, Ades PA. Obesity, body fat distribution, and coronary artery disease. J Cardiopulm Rehabil 2000;20:96-108.

45. Dickinson WP DL, Jortberg BT, Hessler DM, Fernald DH, Fischer L. A cluster randomized trial comparing strategies for translating self-management support into primary care practices. BMC Fam Pract 2018;19:126.

46. Baron RJ. Quality improvement with an electronic health record: achievable, but not automatic. Ann Intern Med 2007;147:549-52.

47. Crosson JC, Etz RS, Wu S, Straus SG, Eisenman D, Bell DS. Meaningful use of electronic prescribing in 5 exemplar primary care practices. Ann Fam Med 2011;9:392-7.
48. Crosson JC, Isaacson N, Lancaster D, et al. Variation in electronic prescribing implementation among twelve ambulatory practices. J Gen Intern Med 2008; 23:364-71.

49. Dickinson WP, Dickinson LM, Jortberg BT, et al. A cluster randomized trial comparing strategies for translating self-management support into primary care practices. J Am Board Fam Med 2019;32:341352.

50. Fernald DH, Jortberg BT, Hessler DM, Wearner R., Dickenson, L.M., Fisher, L, Dickinson, W.P. Recruiting primary care practices for research: reflections and reminders. J Am Board Fam Med 2018; 31:947-951.

51. Dickinson WP, Dickinson LM, Nutting PA, et al. Practice facilitation to improve diabetes care in primary care: a report from the EPIC randomized clinical trial. Ann Fam Med 2014;12:8-16.

52. Harris PA, Taylor R, Thielke R, Payne J, Gonzalez N, Conde JG. Research electronic data capture (REDCap)-a metadata-driven methodology and workflow process for providing translational research informatics support. J Biomed Inform 2009; 42:377-81.

53. Miles MB, Huberman AM, Saldana J. Qualitative data analysis: a methods sourcebook, 3rd ed. Thousand Oaks, CA: SAGE Publications; 2014.

54 Miller WL, Crabtree BF. The dance of interpretation. Thousand Oaks, CA: Sage Publications; 1999.

55. Shetty G, Brownson CA. Characteristics of organizational resources and supports for self management in primary care. Diabetes Educ 2007;33:185s-92s.

56. Lehman WE, Greener JM, Simpson DD. Assessing organizational readiness for change. J Subst Abuse Treat 2002;22:197-209. 